\title{
Fourth order resonance of a high intensity linear accelerator
}

\author{
D. Jeon, ${ }^{1, *}$ L. Groening, ${ }^{2}$ and G. Franchetti ${ }^{2}$ \\ ${ }^{1}$ SNS, Oak Ridge National Laboratory, Oak Ridge, Tennessee 37831, USA \\ ${ }^{2}$ GSI, Darmstadt, Germany \\ (Received 15 January 2009; published 29 May 2009)
}

\begin{abstract}
It is discovered that, for a high intensity beam, the $4 \sigma=360^{\circ}$ (or $4 \nu=1$ ) resonance of a linear accelerator is manifested through the octupolar term of space charge potential when the depressed phase advance per cell $\sigma$ is close to and below $90^{\circ}$ but no resonance effect is observed when $\sigma$ is just above $90^{\circ}$. To verify that this is a resonance, a frequency analysis is performed and a study of resonance crossing from above and from below the resonance is conducted. It is observed that this fourth order resonance is dominating over the better known envelope instability and practically replacing it. The simulation study shows a clear emittance growth by this resonance and its stop band. A proposal to GSI was made to perform an experiment to measure the stop band of this resonance using the UNILAC. The experiment confirmed this resonance and will be published in a separate paper.
\end{abstract}

DOI: 10.1103/PhysRevSTAB.12.054204

PACS numbers: 29.27.Bd, 41.75.- i

\section{INTRODUCTION}

Recently many high intensity linear accelerators (linacs) have been designed or constructed like the SNS (USA) [1], and J-PARC (Japan) [2], or people are trying to increase the intensity of existing linacs such as the UNILAC of GSI (Germany) [3]. For the high intensity linacs, it is the utmost goal to minimize the beam loss of halo particles by avoiding or minimizing contributions of various halo formation mechanisms. One such mechanism is the envelope instability [4]. So far, the high intensity linac design such as the SNS linac has avoided the zero current phase advance $\sigma_{o}=90^{\circ}$ [1] because of the envelope instability.

Until 1998, mismatch was the primarily studied mechanism of halo formation. In late 1998, Jeon found a case of halo formation induced by the $2 \nu_{x}-2 \nu_{y}=0$ resonance from the space charge potential in the ring [5]. Further studies of halo formation and/or emittance growth by space charge and resonances were reported in [6] and space charge coupling resonance studies of the linac were reported in [7]. Besides these, halo formation by nonround beam was reported in [8] and halo formation by rf cavity in [9]. A space charge driven fourth order mode instability of a ring lattice was reported [10]. Various resonance conditions outside of a Kapchinskij-ladimirskij (KV) beam were reported in [11] and various KV beam mode instabilities in [4], where the KV beam itself does not have any nonlinear potential within the beam.

In this paper, we report a discovery of the $4 \sigma=360^{\circ}$ resonance (the equivalent of the $4 \nu=1$ resonance of a circular accelerator) in high intensity linear accelerators

\footnotetext{
*Corresponding author. jeond@ornl.gov
}

that was never reported before and discuss a proposed experiment. For a high intensity linear accelerator, the tune $\nu$ can be defined as $\nu \equiv \sigma / 360^{\circ}$.

\section{THE $4 \sigma=360^{\circ}($ OR $4 \nu=1)$ RESONANCE FOR A HIGH INTENSITY LINAC}

Numerical simulation of a linac is performed with a fairly well matched beam with 50000 to 100000 macroparticles using the PARMILA code with a 3D space charge routine [12]. A space charge grid with $\left(N_{x}=8, N_{y}=8\right.$, $\left.N_{z}=20\right)$ is used for the simulation. A $10 \mathrm{emA}{ }_{40} \mathrm{Ar}^{+10}$ beam with initial $\beta=0.103$ and initial normalized rms emittance $\varepsilon_{x}=\varepsilon_{y}=0.115$ [mm mrad], $\varepsilon_{z}=$ 0.119 [mm mrad] is used for most of the simulations unless specified otherwise. The beam is accelerated to $\beta=0.24$. The space charge phase advance depression is about $-20^{\circ}$. The coupling between the transverse and longitudinal planes is minimal because the depressed longitudinal phase advance is about $10^{\circ}$ well separated from the depressed transverse phase advance. A linac with an focusing-focusing-defocusing-defocusing (FFDD) transverse focusing lattice is used for the simulations. This resonance is observed independent of the choice of the lattice such as FD (focusing quad and defocusing quad) or FFDD.

The study shows that the $4 \sigma=360^{\circ}$ (or $4 \nu=1$ ) resonance occurs when the depressed phase advance $\sigma$ is close to and below $90^{\circ}$ for a high intensity linac just like a ring through the space charge octupole potential for a variety of beams including Gaussian, waterbag, etc. However, no resonance effect is observed when $\sigma$ is slightly above $90^{\circ}$, as shown in Fig. 1. When $\sigma$ is slightly above $90^{\circ}$, we observe no fourfold structure unlike the case with $\sigma$ below $90^{\circ}$ and the emittance growth is very small, which is 


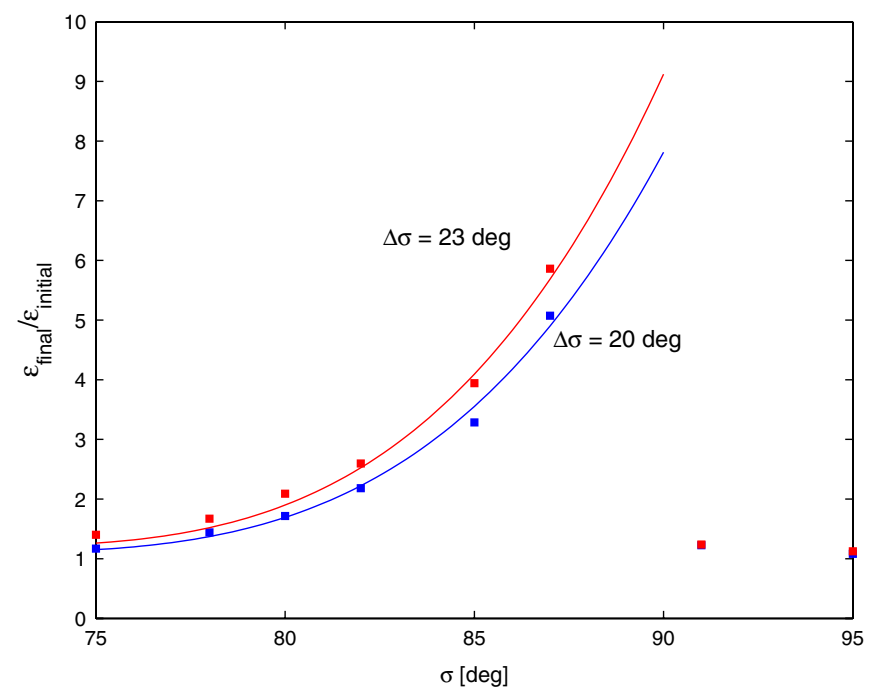

FIG. 1. (Color) Plots of the rms emittance growth vs the depressed phase advance $\sigma$ for two different tune depressions, that is, two different beam current values. There is no resonance effect or emittance growth for $\sigma>90^{\circ}$. An emittance growth of more than $400 \%$ is observed for $\sigma=87^{\circ}$.

due to the tiny initial mismatch. As shown in Fig. 1, the emittance growth increases as $\sigma$ approaches the resonance $\sigma=90^{\circ}$.

One characteristic of this resonance is the behavior difference when we cross the resonance from below and from above the resonance. This is due to the stable fixed points of the resonance. When we cross the resonance from above, the four stable fixed points emerge from the origin and move away, scooping particles from the core (see the bottom plot of Fig. 2). On the other hand, when we cross the resonance from below, stable fixed points move in from far toward the origin. So the particles cannot be captured by the stable fixed points, and they move around the fixed points (the top plot of Fig. 2). Figure 2 shows this behavior difference well, resulting in the beam distribution difference when we cross the $4 \sigma=360^{\circ}$ resonance from below (top plot where $\sigma$ varies from $70^{\circ}$ to $95^{\circ}$ ) and from above (bottom plot where $\sigma$ varies from $100^{\circ}$ to $75^{\circ}$ ).

Depending on the direction to cross the $4 \sigma=360^{\circ}$ resonance, the emittance growth also differs for the same reason. Figure 3 shows the plot of the emittance growth vs a parameter $S \equiv\left(\Delta \sigma / 360^{\circ}\right)^{2} /\left(d \sigma / d n / 360^{\circ}\right)$ [13], where $\Delta \sigma$ is the tune spread (proportional to the stop band width of the resonance) and $d \sigma / d n$ is the phase advance change per cell. Emittance growth for two different groups is shown when we cross the $4 \sigma=360^{\circ}$ resonance from above the resonance (downward crossing) and from below (upward crossing). We observe that the emittance growth is mostly linear for both cases except for $S>2$. A large value of $S$ means slow resonance crossing or wide resonance stop band.

Another characteristic of the resonance is the existence of a resonant frequency component. Because of the fixed
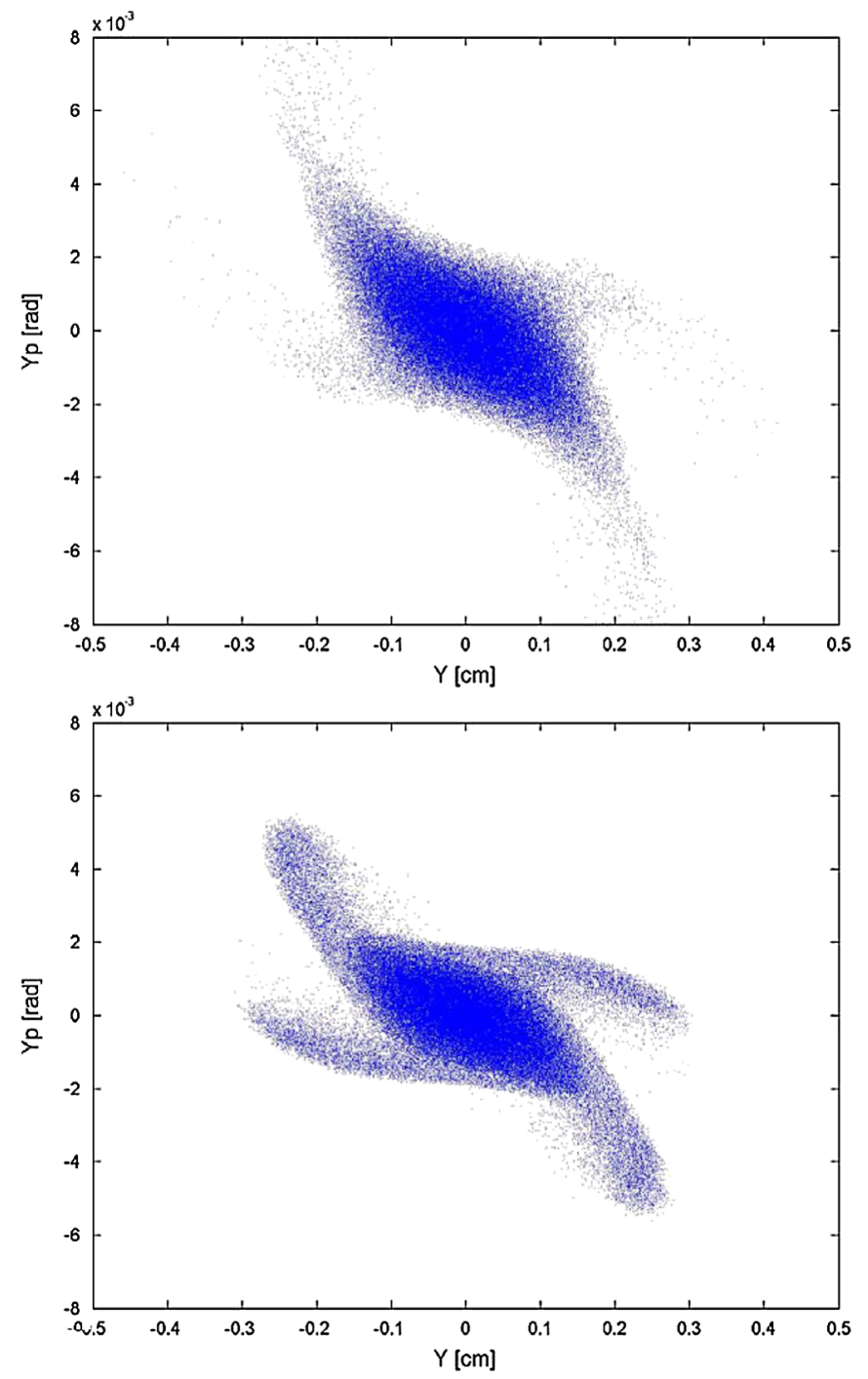

FIG. 2. (Color) Beam distribution plots when we cross the $4 \sigma=$ $360^{\circ}$ resonance from below the resonance (top plot) and when crossing the resonance from above (bottom plot). The top plot corresponds to $\sigma=87^{\circ}$ and the bottom plot corresponds to $\sigma=$ $85^{\circ}$.

points of the resonance, some particles have the same transverse oscillation frequency as the driving frequency of the $4 \sigma=360^{\circ}$ resonance. A Fourier analysis is performed on the 2nd moment $\left\langle X^{2}\right\rangle$ (or $\left\langle Y^{2}\right\rangle$ ) of the beam distribution along the linac when the depressed phase advance $\sigma$ of the linac lattice is $75^{\circ}, 85^{\circ}$, and $92^{\circ}$, and a clear $4 \sigma=360^{\circ}$ resonance peak is observed at the oscillation tune of individual particles (number of transverse oscillation particles made within one cell) with a value of $0.25\left(=90^{\circ} / 360^{\circ}\right)$ for the cases with $\sigma<90^{\circ}$, as shown in Fig. 4. We observe no resonance peak at the tune of 0.25 when $\sigma=92^{\circ}$ just above the resonance as is expected for $\sigma>90^{\circ}$. For each $\sigma$ value, we keep the depressed phase advance $\sigma$ pretty constant throughout the lattice. 


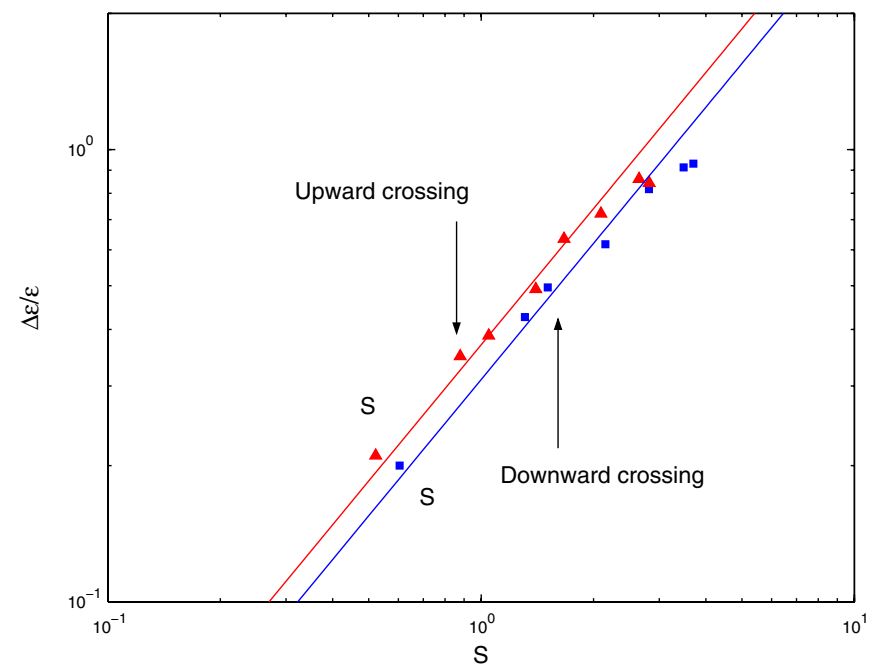

FIG. 3. (Color) Plot of emittance growth when we cross the $4 \sigma=360^{\circ}$ resonance from above (downward crossing) and from below (upward crossing). The emittance growth is proportional to $S$ up to around $S=2$. Here $S=$ $\left(\Delta \sigma / 360^{\circ}\right)^{2} /\left(d \sigma / d n / 360^{\circ}\right)$, where $\Delta \sigma$ is the tune depression (proportional to the stop band width of the resonance) and $d \sigma / d n$ is the phase advance change per cell.

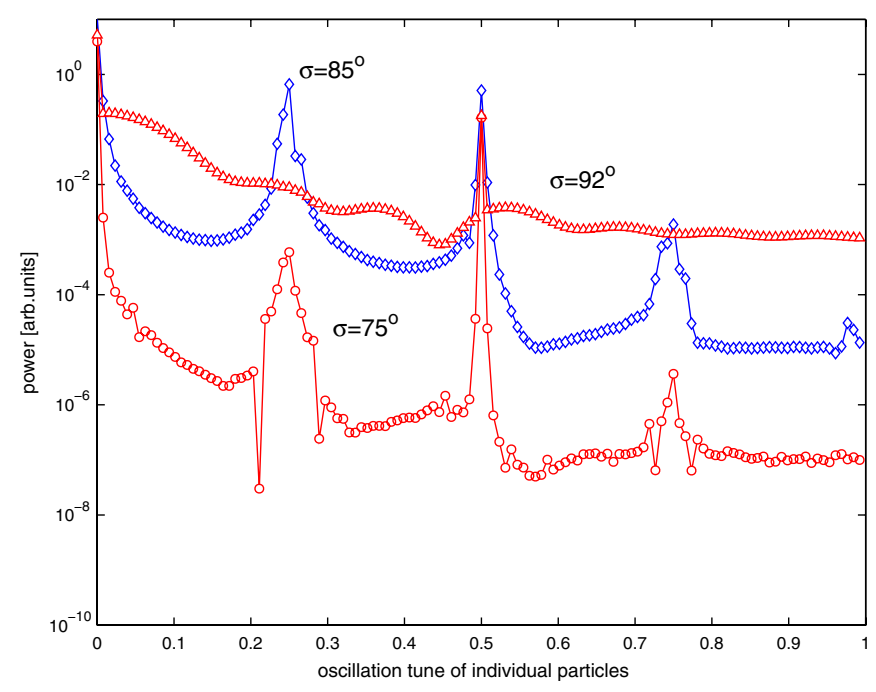

FIG. 4. (Color) Power spectrum plot of the frequency analysis on the 2nd order moment when the depressed phase advance $\sigma$ of the linac lattice is $75^{\circ}, 85^{\circ}$, and $92^{\circ}$. The oscillation tune is defined as the number of transverse oscillations that individual particles make over one cell. When particles are trapped by the fourth order resonance stable fixed points, they make 0.25 turn over one cell. For the two cases with $\sigma<90^{\circ}$, we see a clear $4 \sigma=360^{\circ}$ resonance peak at the tune of $0.25\left(90^{\circ} / 360^{\circ}\right)$, while no resonance peak is observed for the case with $\sigma>90^{\circ}$.

\section{ENVELOPE INSTABILITY}

High intensity linac design has avoided $90^{\circ}$ phase advance because of the well-known envelope instability. Our study indicates that the $4 \sigma=360^{\circ}$ (or $4 \nu=1$ ) resonance

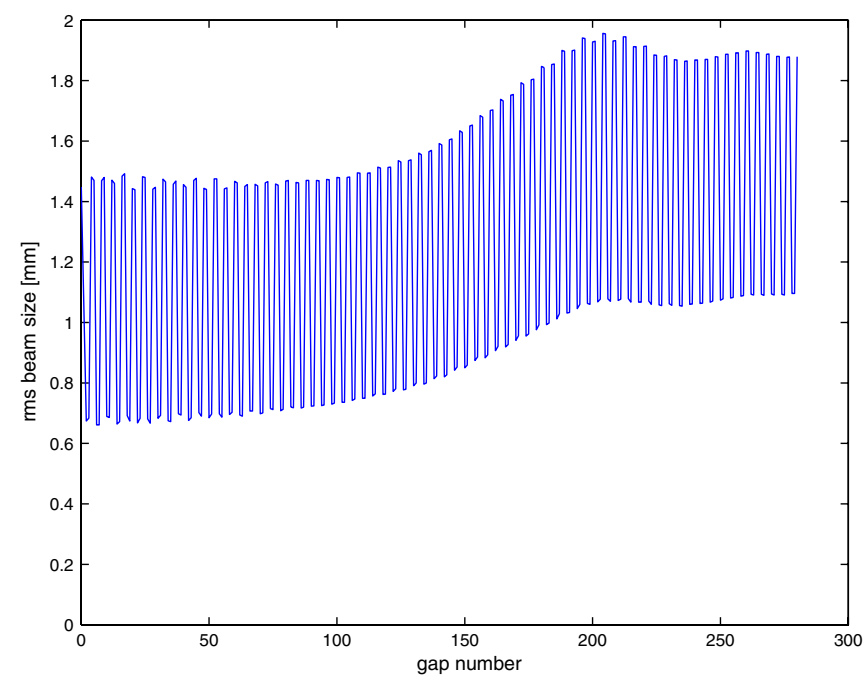

FIG. 5. (Color) Plot of rms beam size vs gap number. The rms beam size is shown as the beam crosses the $4 \sigma=360^{\circ}$ (or $4 \nu=$ 1) resonance from above ( $\sigma$ varies linearly from $99^{\circ}$ to $75^{\circ}$ ). As we monitor the change in the phase space throughout the crossing, we only observe a smooth development of the fourfold structure due to the resonance as in Fig. 2 and do not see any coherent change of the whole beam. The envelope instability is not observed, which is also indicated by the lack of the erratic change in the beam size. The beam size increase is due to the $4 \sigma=360^{\circ}$ resonance as we observe the evolution of the phase space beam distribution. One cell consists of four gaps due to FFDD transverse focusing.

is dominating over the envelope instability and practically replacing it. We did not observe the envelope instability during the simulation for the phase advance around $90^{\circ}$ as shown in Fig. 5. Figure 5 shows the rms beam size as the beam crosses the $4 \sigma=360^{\circ}$ (or $4 \nu=1$ ) resonance from above, where $\sigma$ varies linearly from $99^{\circ}$ to $75^{\circ}$. Here the beam size increase from about the 100th gap on is due to the resonance and the envelope instability is not observed. The characteristic of the envelope instability is the erratic change in the beam envelope (or rms beam size) and the coherent increase/decrease of the entire beam, not just part of the beam. Instead we see a smooth development of the fourfold structure due to the resonance as shown in Fig. 2, resulting in the change in the rms beam size shown in Fig. 5.

Considering this, it should be stated that the high intensity linac design should avoid $\sigma=90^{\circ}$ phase advance because of the $4 \sigma=360^{\circ}$ (or $4 \nu=1$ ) resonance rather than the better known envelope instability. The effect of the envelope instability can actually be minimized-in theory-by nearly perfect envelope matching, whereas the $4 \sigma=360^{\circ}$ resonance is independent of the rms matching. The solution is to stay outside of $90^{\circ}-\Delta Q \leq \sigma \leq$ $90^{\circ}$, where $\Delta Q$ is the stop band width of the fourth order resonance which depends on space charge tune depression. 


\section{PROPOSAL TO MEASURE THE RESONANCE STOP BAND USING GSI UNILAC}

One of the authors (Jeon) made a proposal to GSI colleagues to measure the stop band of this fourth order resonance in April 2007, and since then significant simulation efforts were undertaken to optimize the conditions to measure the stop band. Simulations were performed to predict the emittance growth at the end of the UNILAC Tank 1 as a function of the zero current phase advance. It should be noted zero current phase advance was used as an independent variable because $\sigma_{x}$ and $\sigma_{y}$ are different for the same zero current phase advance because the initial $x$ and $y$ normalized rms emittances of the real beam are different, $\varepsilon_{x}=0.151$ and $\varepsilon_{y}=0.214 \mathrm{~mm} \mathrm{mrad}$. For instance, $\sigma_{x}=90^{\circ}$ corresponds to $\sigma_{x, o}=109.4^{\circ}$ and $\sigma_{y}=$ $90^{\circ}$ to $\sigma_{y, o}=105.6^{\circ}$. Simulations were done for the real UNILAC machine lattice and the same realistic $7.1 \mathrm{emA}$ ${ }_{40} \mathrm{Ar}^{+10}$ beam with $199 \mathrm{~K}$ macroparticles used for the previous benchmarking effort [14]. This input beam was obtained by reconstructing from the emittance measurement conducted before the UNILAC Tank 1. Figure 6 shows the simulation prediction of the average of the experimental transverse rms emittances $\left(\varepsilon_{x}+\varepsilon_{y}\right) / 2$ at the end of UNILAC DTL Tank 1 vs the zero current phase advance. We observe a clear emittance growth due to the resonance.

The proposed experiment was conducted successfully in December 2008, confirming this simulation prediction to be pretty accurate compared with the experiment result. The experimental result is submitted in a separate paper [15]. Because the UNILAC Tank 1 is relatively short (about 16 cells), maximum emittance growth takes place

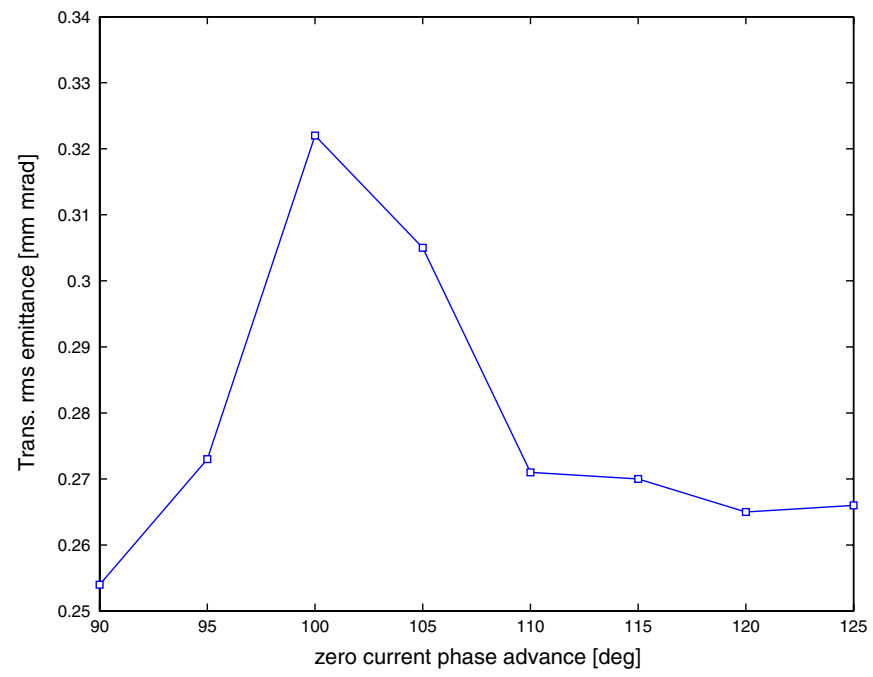

FIG. 6. (Color) Plot of simulation prediction of experiment emittance showing the stop band of the $4 \sigma=360^{\circ}$ resonance at the end of the UNILAC Tank 1. Plotted is the average of normalized rms emittances $\left(\varepsilon_{x}+\varepsilon_{y}\right) / 2$ vs the zero current phase advance of the UNILAC Tank 1.

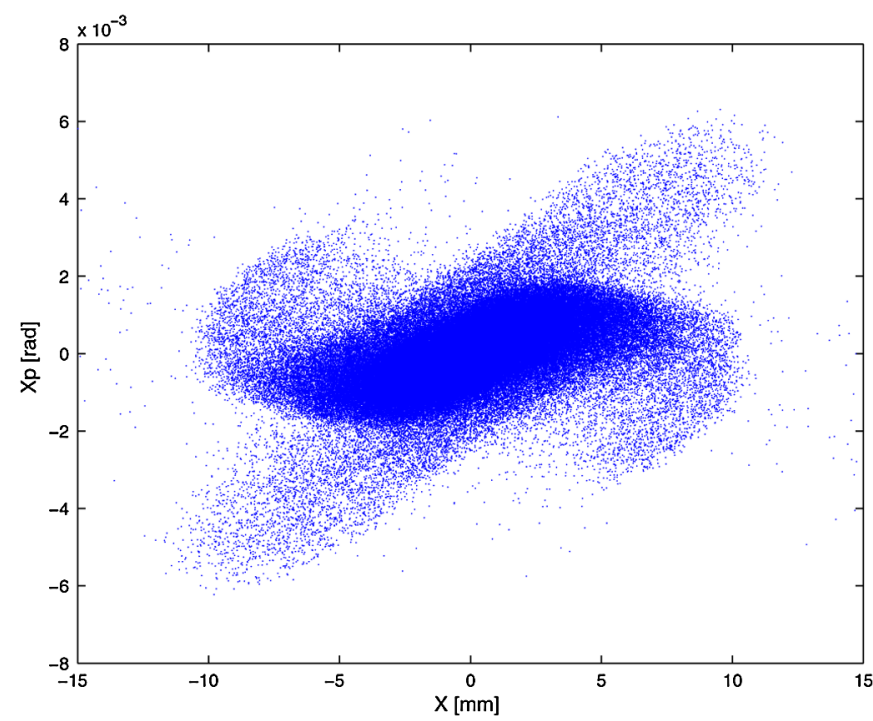

FIG. 7. (Color) Plot of simulated beam distribution in $x$ phase space for $\sigma_{o}=100$ at the end of the UNILAC Tank 1. This shows well the fourfold structure of the $4 \sigma=360^{\circ}$ resonance.

around $\sigma_{o}=100^{\circ}$. Figure 7 shows the simulated beam distribution in $x$ phase space at the exit of UNILAC Tank 1 for zero current phase advance $\sigma_{o}=100^{\circ}$ illustrating well the fourfold structure of the $4 \sigma=360^{\circ}$ resonance. The resonance stop band can be deduced from further simulation after it is confirmed that the simulation agrees well with the experiment.

\section{CONCLUSIONS}

The most important result presented in this paper is the discovery of a fourth order resonance in high intensity linear accelerators that was never reported before. The $4 \sigma=360^{\circ}$ resonance of a linear accelerator (equivalent of the $4 \nu=1$ resonance of a circular accelerator) is manifested through the space charge potential for a high intensity beam when the depressed phase advance $\sigma$ is close to and below $90^{\circ}$ but no resonance effect is observed just above $90^{\circ}$. The frequency analysis study and the study of resonance crossing from above and from below the resonance confirm that the observed phenomenon is the $4 \sigma=$ $360^{\circ}$ (or $4 \nu=1$ ) resonance. It is observed that this fourth order resonance is dominating over the better known envelope instability and practically replacing it. It needs to be rephrased that the high intensity linac design should avoid $90^{\circ}$ phase advance because of the $4 \sigma=360^{\circ}$ ( or $4 \nu=1$ ) resonance rather than the better known envelope instability.

\section{ACKNOWLEDGMENTS}

This work is a result of the collaboration between GSIFAIR and SNS. The authors would like to express their gratitude to Professor I. Hofmann for his advice and comments. One of the authors (D. J.) is grateful for the hospital- 
ity of GSI and the partial support through the EU-FP6 CARE-HIPPI (Contract No. RII3-CT-2003-506395). He also is very grateful for the support of the SNS management. SNS is managed by UT-Battelle, LLC, under Contract No. DE-AC05-00OR22725 for the U.S. Department of Energy.

[1] J. Stovall et al., Proceedings of the Particle Accelerator Conference, Chicago, IL, 2001 (IEEE, New York, 2001), p. 446.

[2] Y. Yamazaki, Proceedings of the 20th Particle Accelerator Conference, Portland, OR, 2003 (IEEE, New York, 2003), p. 576.

[3] W. Barth et al., Proceedings of the 2004 LINAC Conference, Luebeck, Germany (GSI, Luebeck, Germany, 2004), p. 246.

[4] I. Hofmann, L. J. Laslett, L. Smith, and I. Haber, Part. Accel. 13, 145 (1983).

[5] D. Jeon, J. A. Holmes, V. V. Danilov, J. D. Galambos, and D. K. Olsen, Phys. Rev. E 60, 7479 (1999).

[6] G. Franchetti et al., Phys. Rev. ST Accel. Beams 6, 124201 (2003).
[7] G. Franchetti, I. Hofmann, and D. Jeon, Phys. Rev. Lett. 88, 254802 (2002).

[8] D. Jeon, Proceedings of the 2007 Asian Particle Acceleration Conference, Indore, India (RRCAT, Indore, 2007), p. 333.

[9] M. Eshraqi, G. Franchetti, and A. M. Lombardi, Phys. Rev. ST Accel. Beams 12, 024201 (2009).

[10] I. Hofmann, Proceedings of the Workshop on the Use of the Spallation Neutron Source for Heavy Ion Fusion Beam Dynamics Studies, Rutherford Laboratories, U.K. (1981), p. 77, http://accelconf.web.cern.ch/accelconf/.

[11] J-M. Lagniel, Nucl. Instrum. Methods Phys. Res., Sect. A 345, 405 (1994).

[12] H. Takeda and J. Stovall, Proceedings of the Particle Accelerator Conference, Dallas, TX, 1995 (IEEE, New York, 1995), p. 2364.

[13] I. Hofmann and G. Franchetti, Proceedings of the 2007 Particle Accelerator Conference, Albuquerque, New Mexico, 2007 (IEEE, Albuquerque, New Mexico, 2007), p. 3259.

[14] L. Groening, W. Barth, W. Bayer, G. Clemente, L. Dahl, P. Forck, P. Gerhard, I. Hofmann, G. Riehl, S. Yaramyshev, D. Jeon, and D. Uriot, Phys. Rev. ST Accel. Beams 11, 094201 (2008).

[15] L. Groening et al., Phys. Rev. Lett. (to be published). 